\title{
Genomic Survey of ATP-Binding Cassette (ABC) Transporters in Sorghum bicolor (L.) Moench
}

\author{
Olawuyi $\mathrm{OJ}^{1^{*}}$ and Ologidi $\mathrm{CG}^{1,2}$ \\ ${ }^{1}$ Department of Botany, University of Ibadan, Genetics and Molecular Biology Unit, Ibadan, Nigeria \\ ${ }^{2}$ Department of Biological Sciences, Niger Delta University, Wilberforce Island, Amassama, Nigeria
}

${ }^{*}$ Corresponding author: Olawuyi OJ, Department of Botany, University of Ibadan, Genetics and Molecular Biology Unit, Ibadan, Nigeria, Tel: +2348168223985, E-mail: olawuyiodunayo@yahoo.com

Citation: Olawuyi OJ, Ologidi CG (2018) Genomic Survey of ATP-Binding Cassette (ABC) Transporters in Sorghum Bicolor (L.) Moench. J Plant Sci Crop Protec 1(1): 102. doi: 10.15744/2639-3336.1.102

Received Date: February 15, 2018 Accepted Date: April 30, 2018 Published Date: May 02, 2018

\begin{abstract}
ATP-binding cassette $(\mathrm{ABC})$ transporters are the largest and most ancient family of transmembrane proteins with representatives in all phyla from prokaryotes to humans. Systematic searches were performed using PSI-Blast program on NCBI to identify sorghum $\mathrm{ABC}$ transporters. The identified proteins were subjected to phylogenetic and domain topology analyses and were classified and named according to the Human Genome Organisation (HUGO) system of classification and unified plant nomenclatural system. As part of the survey, the subcellular location, presence of signal peptide and physicochemical parameters were determined. The results from the genomic survey showed that $100 \mathrm{ABC}$ transporters were identified and classified as $\mathrm{ABCB}, \mathrm{ABCC}, \mathrm{ABCD}, \mathrm{ABCE}, \mathrm{ABCF}$, and $\mathrm{ABCI}$. Seventy one proteins encode intrinsic membrane proteins containing nucleotide-binding and transmembrane domains (NBD and TMD) and 28 encode proteins without TMDs. The Sorghum bicolor ABC transporter family consists of 61 full-size molecules, 9 halfsize molecules, 3 three-quarter-size molecules, and 26 quarter-size molecules. One of the proteins (GI-992164873) has neither TMD nor NBD. A majority of the proteins are located in the plasma membrane. Signal peptide was observed in SbABCB11, SbABCB34, SbABCC4, SbABCC21, and SbABCI3. The proteins had varying values of physicochemical parameters.
\end{abstract}

Keywords: Sorghum bicolor; ABC transporters; proteins; Genome

\section{Introduction}

Sorghum bicolor ranks fifth most important cereal crop in the world, and is the first plant of African origin whose genome had been sequenced. A large amount of the grains in the African continent, for instance 85 to $95 \%$ produced in Nigeria, are consumed as food; a smaller amount is used in brewery and as industrial raw materials [1].

ATP-binding cassette $(\mathrm{ABC})$ transporters are largest members of diverse proteins constituting the most ancient families with representatives in all phyla from prokaryotes to humans [2]. They are transmembrane proteins that use the energy of adenosine triphosphate (ATP) binding and hydrolysis to perform certain biological processes including translocation of various solutes across membranes and non-transport-related processes such as DNA repair and translation of RNA. They transport wide ranging substances across extra- and intracellular plant membranes, including metabolic products, coating and supportive materials, defense molecules, and plant hormones that modulate the overall physiology, development, and response to biotic and abiotic stresses [3]. Thus arabidopsis ABC transport proteins such as ABCG12, ABCG11, ABCG13 have been shown to play roles in movement of cuticular lipids such that the corresponding mutants exhibits negative phenotypic and physiological aberrations [4-8]. In addition, ABC transporters including AtABCG26 and OsABCG15 have been reported to have important functions in depositing sporopollenin, which is a major component of the outer exine layer of plant spores and pollen [3,9-11]. AtABCG9 and AtABCG31 play important roles in pollen coat maturation [12].

The ABCG transporters NpPDR1/NpABC1 and NtPDR1 are involved in responds to pathogenic attacks by responding to levels of sclareol and jasmonate [13,14]. Similarly, the Coptis japonica ABCB1/MDR1 and ABCB2 are involved in plant defense by catalyzing the import of berberine, a potent antimicrobial compound $[15,16]$. ABC proteins also transport cytokinins, which facilitate communication between the shoot and root systems, abscisic acid that mediates tolerance to stress and inhibition of seed germination, auxin (indole-3-acetic acid, IAA and indole 3-butyric acid, IBA) that regulates growth and tropism, and strigolactone that is involved in plant-microbe interactions and control of shoot branching [17-25]. Furthermore ABC proteins mediate transport of metabolic intermediates and peroxisomes, which supply the required energy for germination of oil-hiving away seeds being the 
site of $\beta$-oxidation through which fatty acids are degraded, phytate (inositohexakisphosphate), a storage form of phosphate, heavy metals such as arsenic, cadmium, and mercury, and membrane lipid metabolites [26-33].

Proteins are classified as $\mathrm{ABC}$ transporters based on sequence homology to prototypes, phylogeny, organization of their ATPbinding cassette $(\mathrm{ABC}$ ) domains, and presence of $\mathrm{ABC}(\mathrm{C})$ motif (also known as C motif or LSGGQ motif, (LIVMFY)S(SG)GX 3(RKA)(LIVMYA)X(LIVFM) [34,35]. Plant genomes contain a plethora of ABC transporters with a few of them experimentally characterized [20]. Due to availability of resources for conducting genetic and molecular studies, Arabidopsis thaliana and Oryza sativa have received a great deal of attention allowing for experimental characterisation of several of their ABC transporters [36,37]. But only auxin transport protein (PGP1) has been experimentally described in Sorghum bicolor [38]. Therefore undertaking a genomic survey of Sorghum bicolor ABC transporters with the intent of producing an inventory of the proteins will provide an opportunity for experimentally characterising more $\mathrm{ABC}$ transport proteins from sorghum. Besides genomic survey of the crop's $\mathrm{ABC}$ transporters has not been carried out despite being the first plant of African origin whose genome had been sequenced [39].

\section{Materials and Methods}

\section{Identification of ABC Transporters in Sorghum bicolor}

Arabidopsis thaliana ABC protein sequences were retrieved from the Arabidopsis Information Resource (TAIR) database (http:// www.arabidopsis.org/browse/genefamily/ABC_merged.jsp). Using the default search parameters of NCBI's Position-Specific Iterated Basic Local Alignment Search Tool (PSI-BLASP) program (http://ncbi.nlm.nih.gov/blast), five position-specific iterative searches for S. bicolor putative/hypothetical ABC transporters were performed with Arabidopsis ABC transporter protein sequence as queries [40]. The PSI-BLASP protein-protein search program is an iterative profile method for identifying conserved patterns in BLAST results [41]. The significant threshold for selection of sequences was score of 400 with E value of e-120 [37].

\section{Domain, Motif, Homology, and Phylogenetic Analyses}

The selected sequences were subsequently subjected to domain and motif analysis-presence of $\mathrm{ABC}$ domains and $\mathrm{ABC}(\mathrm{C})$ signature motifs-using default search parameters of PROSITE (http://prosite.expasy.org/), Conserved Domain Database at NCBI (http:// ncbi.nlm.nih.gov/Structure/cdd/wrpsb.cgi) and TMHMM 2.0 (http://www.cbs.dtu.dk/services/TMHMM/) [34,35,42-44]. The domain features-forward or reverse orientation, number and size (full, three-quarter, half, and one-quarter) - were then noted. For homology analysis, prototypes of $\mathrm{ABC}$ subfamilies (human $\mathrm{PDR} / \mathrm{ABCB} 1$ and $\mathrm{AtABCB} 1$ for $\mathrm{ABCB}$ subfamily; human $\mathrm{ABCC} 1$ and ABCC2 for ABCC subfamily; yeast PXA1 and PXA2 for ABCD subfamily; yeast RLI for ABCE subfamily; and GCN1/AtABCF1 and GCN2/AtABCF2 for ABCF subfamily) were aligned against the selected S. bicolor ABC protein sequences by using default search parameters of blastp [45-49]. Furthermore, phylogenetic analyses were performed using neighbor joining and maximum likelihood methods with 1000 bootstrap replicates after conducting multiple sequence alignment of A. thaliana and S. bicolor ABC transporter sequences with ClustalW alignment tool [50]. Phylogenetic trees were constructed and viewed with MEGA7 and RAxM softwares programs from which similar results were obtained and only trees produced by neighbor joining method are presented and discussed herein $[51,52]$.

\section{Classification of Sorghum bicolor ABC Transporters into Subfamilies}

The Sorghum bicolor ABC transporters identified were classified into one of six ABC subfamilies (ABCB, ABCC, ABCD, ABCE, $\mathrm{ABCF}$, and $\mathrm{ABCI}$ ) based on their domain features: half- and full-sized forward domain topology for ABCB subfamily; threequarter- and full-sized forward topology for ABCC subfamily; half- and full-sized forward orientation for ABCD subfamily; halfsized NBD-only domain topology for subfamilies ABCE and ABCF, and one-quarter-sized bacterial-like domain for subfamily $\mathrm{ABCI}[20,36,45]$. Other conditions used for classifying the proteins were homology to prototype for each subfamily and clustering pattern on phylogenetic tree.

\section{Orthology Analysis}

Analysis for sequences orthologous to sorghum ABC transporters were performed using default settings of Smart Blast (https:// blast.ncbi.nlm.nih.gov/smartblast/). The criteria for selecting orthologous sequences were percent identity of at least $70 \%$, 'E' value of 0.0 , and experimental function characterization.

\section{Results and Discussion}

\section{Identification and Classification of ABC Transporters in Sorghum bicolor}

Systematic BLAST searches of the Sorghum bicolor proteome with amino acid sequences of $A$. thaliana ABC transporters as queries identified $100 \mathrm{ABC}$ transporters in S. bicolor that contained at least one ABC signature (Table S1). The criteria for selection of the sequences were presence of TMDs, NBDs and C motif (LSGGQ). Amongst the S. bicolor ABC transporters identified, 72 encode intrinsic membrane proteins and 28 encode proteins without TMDs. The Sorghum bicolor ABC transporter family consists of 61 full-size molecules, 9 half-size molecules, 3 three-quarter-size molecules, and 26 quarter-size molecules. One of the proteins (GI- 


\section{3) has neither TMD nor NBD.}

None of the proteins showed homology to human ABCA1 and ABCA4 proteins, which are prototypic members of ABCA subfamily. Thus, no ABCA protein was identified in S. bicolor. Similarly, no S. bicolor ABC transporter protein was placed in the ABCG subfamily because they all lack the characteristic reverse domain orientation (that is, NBD-TMD) of the subfamily. Thus, the $S$. bicolor $\mathrm{ABC}$ transporters were classified into 6 subfamilies of $\mathrm{ABCB}, \mathrm{ABCC}, \mathrm{ABCD}, \mathrm{ABCE}, \mathrm{ABCF}$, and $\mathrm{ABCI}$.

Forty (40) proteins of $S$. bicolor $\mathrm{ABC}$ transporters were classified as $\mathrm{ABCB}$ proteins because of their homology to the human $\mathrm{ABCB} 1$, which is a prototype member of ABCB proteins. S. bicolor ABCB transporters consist of 5 half-sized members (TAP/ HMT), 2 three-quarter-sized members, and 33 full-sized members (MDR/PGP) (Table S1). Twenty six (26) S. bicolor ABC transporters belong to ABCC subfamily (Table S1). They showed homology to the human HsMRP1 and mouse cMOAT proteins which are prototypes for ABCC subfamily. ABCC transporters of S. bicolor consists of 2 three-quarter sized members and 24 full-sized members. Three (3) proteins were placed in ABCD subfamily because of their homology to the yeast Pxalp and Pxa2p proteins which are the prototypic members of ABCD subfamily. The 3 proteins have a forward orientation and full-size domain structure (Table S1).

Members of ABCE and ABCF subfamily have a half-sized domain structure and are soluble proteins because they lack transmembrane domain [36]. Five (5) ABC transporters were classified as ABCE and ABCF because of their domain structure. One of the proteins showed homology to yeast Rlilp, which is a prototype for ABCF family. The other four did not show homology to Rlilp and were classified as ABCE transporters (Table S1). Twenty six (26) S. bicolor ABC transporters were identified to having a single NBD or TMD (Table S1). Twenty three (23) of the proteins have a single NBD and 3 (SbABCI22, SbABCI1, SbABCI6) have a single TMD. One protein (SORBI_006G155700) with GI number 992164873 has neither NBD nor TMD but has a C motif that is characteristic of ABC transporters. Thus it was not placed in any of the subfamilies but declared as undefined (Table S1).

Plant genomes code for over $100 \mathrm{ABC}$ transporters [20]. Thus the $100 \mathrm{ABC}$ transporters identified in Sorghum bicolor is in accordance with the plethora of $\mathrm{ABC}$ transporters that have been reported from other plants including Arabidopsis thaliana, Oryza sativa, and Vitis vinifera $[36,37,53]$ (Table S1). However, the total number of the proteins identified in S. bicolor are less than those identified in A. thaliana, Oryza sative, and Vitis vinifera which contain 130,121, 135, respectively [36,37,53]. This variation in number of $\mathrm{ABC}$ transporters in plants is a pointer that there is likely no direct proportional relationship of number of $\mathrm{ABC}$ transporters with genome size and chromosome number. This is further corroborated by the presence of $49 \mathrm{ABC}$ transporters in humans whose genome size and chromosome number are 3,234.83 Mb and 46, respectively [3,54,55]. The large number of $\mathrm{ABC}$ transporters observed in Sorghum bicolor and reported in other plants is rather due to their sessile nature, multicellularity and an across-the-board capacity for synthesising secondary chemicals [56].

Sorghum bicolor genome contains less number of proteins with intrinsic transmembrane domain (Table S1) than A. thaliana, O. sativa and $V$. vinifera which contain 104, 108 and 120, respectively [36,37,53]. However, the Sorghum bicolor genome contains more proteins lacking TMD than A. thaliana, O. sativa, and V. vinifera, which have 18, 9 and 15, respectively [36,37,53]. In addition, the Sorghum bicolor genome contains more full-sized molecules than A. thaliana, which has 43 . The ABC transporters from $V$. vinifera and O. sativa with full size topology of 79 and 72 are higher than the proteins from Sorghum bicolor [37,53]. The half-sized molecules identified in Sorghum bicolor are far less than those identified in A. thaliana, O. sativa and Vitis vinifera, which have 50,41 and 41 , severally $[36,37,53]$. However, the number of quarter-sized molecule in Sorghum bicolor is more than the numbers found in A. thaliana, O. sativa and Vitis vinifera, whose genome contain 17,10 and 6, severally $[36,37,53]$.

Unlike $\mathrm{ABC}$ transporters from A. thaliana, $O$. sativa and Vitis vinifera which were classified into 8 subfamilies of $\mathrm{ABCA}$, $\mathrm{ABCB}$, $\mathrm{ABCC}, \mathrm{ABCD}, \mathrm{ABCE}, \mathrm{ABCF}, \mathrm{ABCG}$, and $\mathrm{ABCI}$, the sorghum $\mathrm{ABC}$ transporters were classified into 6 subfamilies of $\mathrm{ABCB}$, $\mathrm{ABCC}, \mathrm{ABCD}, \mathrm{ABCE}, \mathrm{ABCF}$, and ABCI [36,37,55]. The ABCB subfamily of sorghum contains both full-size and half-size molecules like in A. thaliana, O. sativa and Vitis vinnifera. But Sorghum bicolor has 33 full-size molecules (that is, MDR/PGP) in the ABCB subfamily which is more than the members of the subfamily in A. thaliana, O. sativa and Vitis vinifera which have 21, 24, and 19, severally. The observation of 5 half size molecules of ABCB proteins in Sorghum bicolor genome shows that A. thaliana, O. sativa and Vitis vinifera contain slightly higher number of half size molecules of 7, 6, and 6, respectively.

The number of ABCC (MRP) proteins identified in Sorghum bicolor is the same as those identified in Vitis vinifera with both plants having 26 members belonging to the ABCC subfamily [53]. But the numbers of ABCC proteins in A. thaliana and O. sativa, which are respectively 15 and 17 are lower than that in Sorghum bicolor [36,37]. The S. bicolor and O. sativa genomes have the same number of ABCD proteins with both containing 3 members [37]. The members of the ABCD subfamily is slightly lower in A. thaliana and $O$. sativa, which have 2 and 1 , severally.

$\mathrm{ABCI}$ and ABCC subfamily of Sorghum bicolor ABC transporters have the highest number with both containing 26 proteins. The members of S. bicolor ABCI proteins is higher in number compared to the members of ABCI proteins in the genomes of $A$. thaliana, $O$. sativa and $V$. vinifera which respectively contain 15,10 , and $6[36,37,53]$. Three members of the ABCI subfamily in Sorghum bicolor have a transmembrane domain (TMD) while those in A. thaliana are 7 [36]. Twenty three (23) members of the Sorghum bicolor ABCI subfamily have a nucleotide binding domain while A. thaliana genome has 10 members [36]. 


\section{Phylogeny of Sorghum bicolor ABC Transporters}

The members of the subfamilies generally clustered tightly with each other (Figure 1). Members of the ABCB subfamily clustered together tightly but two members, SbABCB15 and SbABCB14, clustered with SbABCC8, SbABCC20, SbABCC24, and SbABCC2 which are members of ABCC subfamily. In like manner, members of ABCC subfamily clustered tightly with each other but SbABCC16 and SbABCC12 formed a tight cluster with SbABCF1 while SbABCC3 grouped with members of ABCE and ABCF. Members of ABCI subfamily also clustered together tightly. However, a tight clustering of SbABCI16 with SbABCE4 and SbABCI22 with SbABCB17 were observed. The unclassified ABC transporter SORBI_006G155700 (GI: 992164873 ) formed a tight cluster with SbABCI8, which is a member of ABCI subfamily.

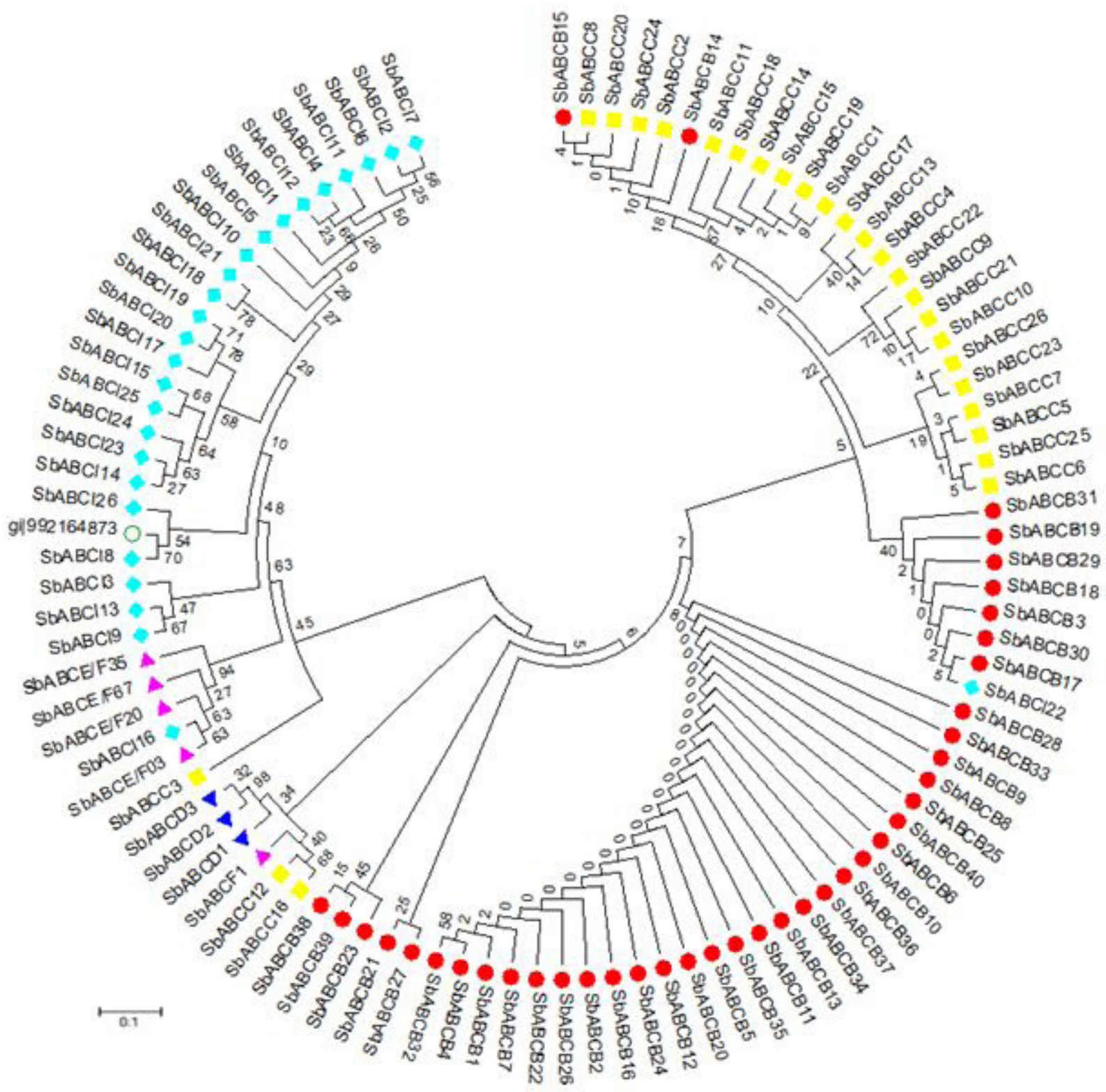

Figure 1: Phylogenetic tree of S. bicolor ABC proteins.
Members of ABCB subfamily.
$\triangle$ Members of ABCD subfamily.
$\triangle$ Members of $\mathrm{ABCE}$ and $\mathrm{ABCF}$ subfamilies
Members of ABCC subfamily. GI: 992164873

The generally very close clustering of members of each subfamily is as a result of the high level of similarity shared, which is due to the conserved $\mathrm{C}$ motif of the nucleotide binding domain (NBD) (Figure 1). The general tight clustering pattern observed in Figure 1 indicates that the nomenclatural and classification schemes adopted for Sorghum bicolor ABC transporters were effective schemes for delineating the proteins [36]. Similar clustering patterns of ABC transporters have been observed in A. thaliana, $O$. sativa, Vitis vinifera and Lotus japonicus [36,37,53,57]. 
Journal of Plant Sciences and Crop Protection

The clustering of SbABCB14 and SbABCB15 with SbABCC8, SbABCC20, SbABCC24 and SbABCC2, which are members of the $\mathrm{ABCB}$ subfamily, is due to the similarity in the C motif sequences; LSGGQKQRIQLSRAL and LSGGQKQRIQLARAV (Figure 1). Notwithstanding, SbABCB14 and SBABCB15 were placed in the ABCB subfamily because of their homology to the human ABCB1, which is a prototypic member of $\mathrm{ABCB}$ subfamilies. In addition, similarity in $\mathrm{C}$ motif sequences accounts for the grouping of SbABCC16 and SbABCC12 with SbABCF1 (Figure 1); LSGGQRARLALARAL, FSVGQRQLLCLARAI, FSGGWKMRMSLGKIL, LSGGEKARLAFCKFM. The same reason can also be given for the clustering of SbABCC3 with members from other subfamilies. Nonetheless, SbABCC16, SbABCC12, and SbABCC3 were classified as ABCC transporter proteins because they showed homology to the human HsMRP1 and mouse cMOAT proteins which are prototypes of ABCC subfamily.

The tight clustering of SORBI_006G155700 (GI: 992164873) with SbABCI8; SbABCI16 with SbABCE4; SbABCI22 with SbABCB17; and SbABCF1 with SbABCC12 and SbABCC16 (Figure 1) can be attributed to a high similarity in their C motif sequences.

\section{Phylogeny of Sorghum bicolor and Arabidopsis thaliana ABCB Subfamilies}

The result from phylogeneitc analysis of ABCB subfamily proteins from Sorghum bicolor and Arabidopsis thaliana is shown in Figure 2. The protein sequences from A. thaliana grouped closely with those from S. bicolor with bootstrap values from 23 to 100 . This is due to the high level of similarity between proteins from both plants. Eight clusters were formed between protein sequences from A. thaliana and S. bicolor. A cluster was formed around AtABCB15, AtABCB17, AtABCB16, AtABCB18, and AtABCB22. AtABCB8 (P-glycoprotein 8) formed a cluster with SbABCB20, SbABCB28, and SbABCB2. There was also clustering of AtABCB2 and AtABCB10 with SbABCB4 and SbABCB32. Another clustering observed in the phylogenetic tree was AtABCB1, AtABCB13, AtABCB14, and AtABCB19 with SbABCB8, SbABCB25 (PGP1), SbABCB6, and SbABCB5 (Figure 1). Furthermore, a cluster group was formed by AtABCB3, AtABCB5, AtABCB11, AtABCB12, AtABCB4, and AtABCB21 with SbABCB9 and SbABCB33. SbABCB1 and AtABCB27 tightly clustered together. A close clustering group was also formed by AtABCB26 with SbABCB38 and SbABCB39.

Two empirically characterised ABCB proteins, AtABCB1 (alias AtPGP1) and SbABCB25 (alias SbPGP1) which are involved in auxin efflux transport formed a cluster [38,58] (Figure 1). Thus the tight clustering of SbABCB8 with SbPGP1 and AtPGP1 is an indication that $\mathrm{SbABCB} 8$ functions in auxin efflux transport. Other proteins that are likely functioning in auxin efflux transport are SbABCB6 and SbABCB9, which formed a tight clustering with AtABCB19 (alias AtPGP19, MRP11) [58]. Similarly, the function of $\mathrm{SbABCB} 1$ can be inferred from the function of AtABCB27 (alias aluminium tolerance-related ABC transporter, AtTAP2), which is possibly involved in redistributing internalized aluminium and may mediate vacuolar sequestration of a metal complex [36].

\section{Phylogeny of Sorghum bicolor and Arabidopsis thaliana ABCC Subfamilies}

The result from phylogenetic analysis of ABCC subfamily proteins from Sorghum bicolor and Arabidopsis thaliana is presented in Figure 3. The protein sequences from A. thaliana grouped closely with those from S. bicolor with bootstrap values between 39 and 100. Eight clusters were formed between proteins from A. thaliana and S. bicolor. AtABCC5 and SbABCC1 formed a cluster group. AtABCC15 and AtABCC9 clustered with SbABCC22, SbABCC10, SbABCC21, and SbABCC9. AtABCC7, AtABCC6, AtABCC3 grouped with SbABCC23, SbABCC7, SbABCC26, SbABCC6, SbABCC25, and SbABCC5. Other groups comprise AtABCC11, AtABCC12, AtABCC1, AtABCC2, and SbABCC3; AtABCC13, SbABCC12, and SbABCC16; SbABCC8, SbABCC20, SbABCC2, SbABCC24, AtABCC4, and AtABCC14; AtABCC10, SbABCC14, SbABCC15, SbABCC19, SbABCC11, and SbABCC18; AtABCC8, SbABCC4, SbABCC17, and SbABCC13.

Based on clustering with ABC transporters from Arabidopsis thaliana, the function of SbABCC23, SbABCC7, SbABCC26, SbABCC6, SbABCC25, and SbABCC5 can be inferred as conjugate and heavy metal (for example, cadmium) transporters because AtABCC7 and AtABCC3 (alias MRP7) are pumps for glutathione S-conjugates and AtABCC3 (alias MRP3) transports heavy metals such as cadmium [59] (Figure 3).

In like manner, SbABCC23, SbABCC7, SbABCC26, SbABCC6, SbABCC25, SbABCC5 which formed a cluster with AtABCC7, AtABCC3, and AtABCC6 are deduced to function as pumps for glutathione S- conjugates. This is because AtABCC7 and AtABCC3 are pumps for glutathione S-conjugates [59].

The S. bicolor genome observed to having 4 members of ABCE subfamily is the plant genome with the highest number of ABCE proteins when compared with $A$. thaliana, $O$. sativa and $V$. vinifera whose inventory of ABC transporters have been published $[36,37,53]$ (Table S1). However, only 1 S. bicolor ABC transporter protein was classified as ABCF protein (Table S1) as opposed to 5 in both Arabidopsis thaliana and V. vinifera and 4 in O. sativa [36,37,53].

Other Sorghum bicolor ABCC proteins that are deduced to function as pumps for glutathione S-conjugate are SbABCC13, SbABCC17, and SbABCC4; SbABCC14, SbABCC15, SbABCC19, SbABCC11, and SbABCC18; SbABCC16 and SbABCC12 which formed clusters with AtABCC8, AtABCC10, and AtABCC13, respectively.

The cluster group observed between S. bicolor ABC transporter proteins, SbABCC2, SbABCC20, SbABCC8, SBABCC24 and A. thaliana proteins AtABCC14 and AtABCC4 suggests that the Sorghum bicolor proteins are functioning in pumping of glutathione 


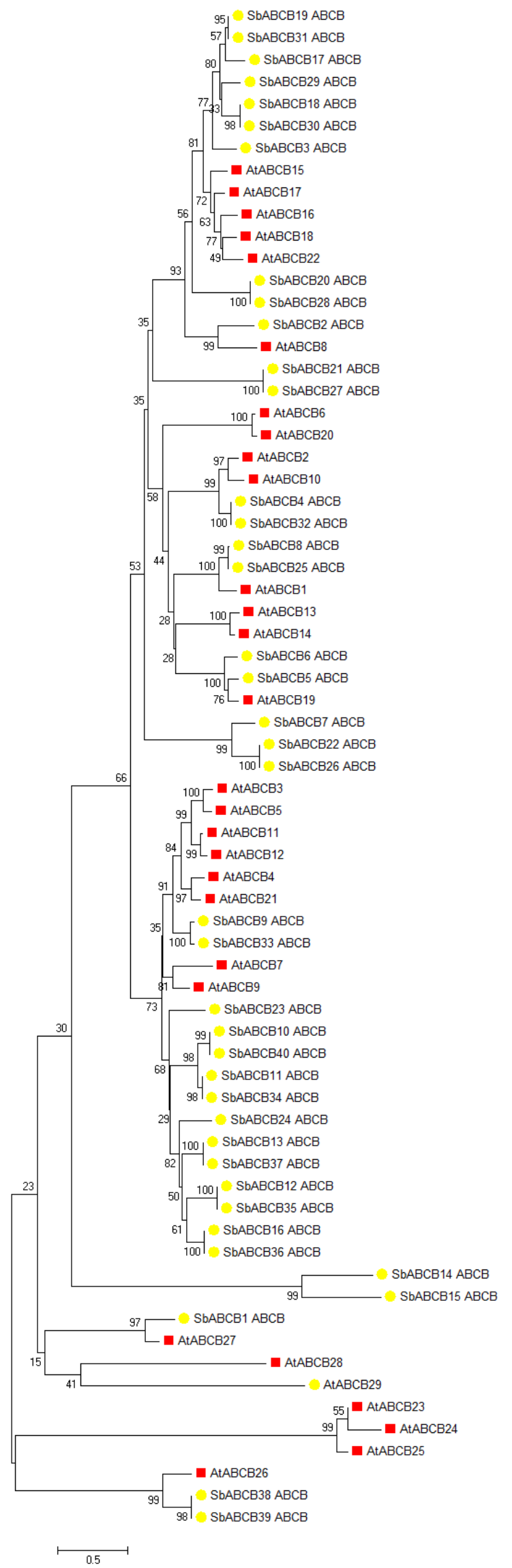

Figure 2: Phylogenetic tree of $\mathrm{ABCB}$ protein sequences from S. bicolor and A. thaliana

$\square$ S. bicolor $\mathrm{ABCB}$ proteins

$\square$ A. thaliana $\mathrm{ABCB}$ proteins 


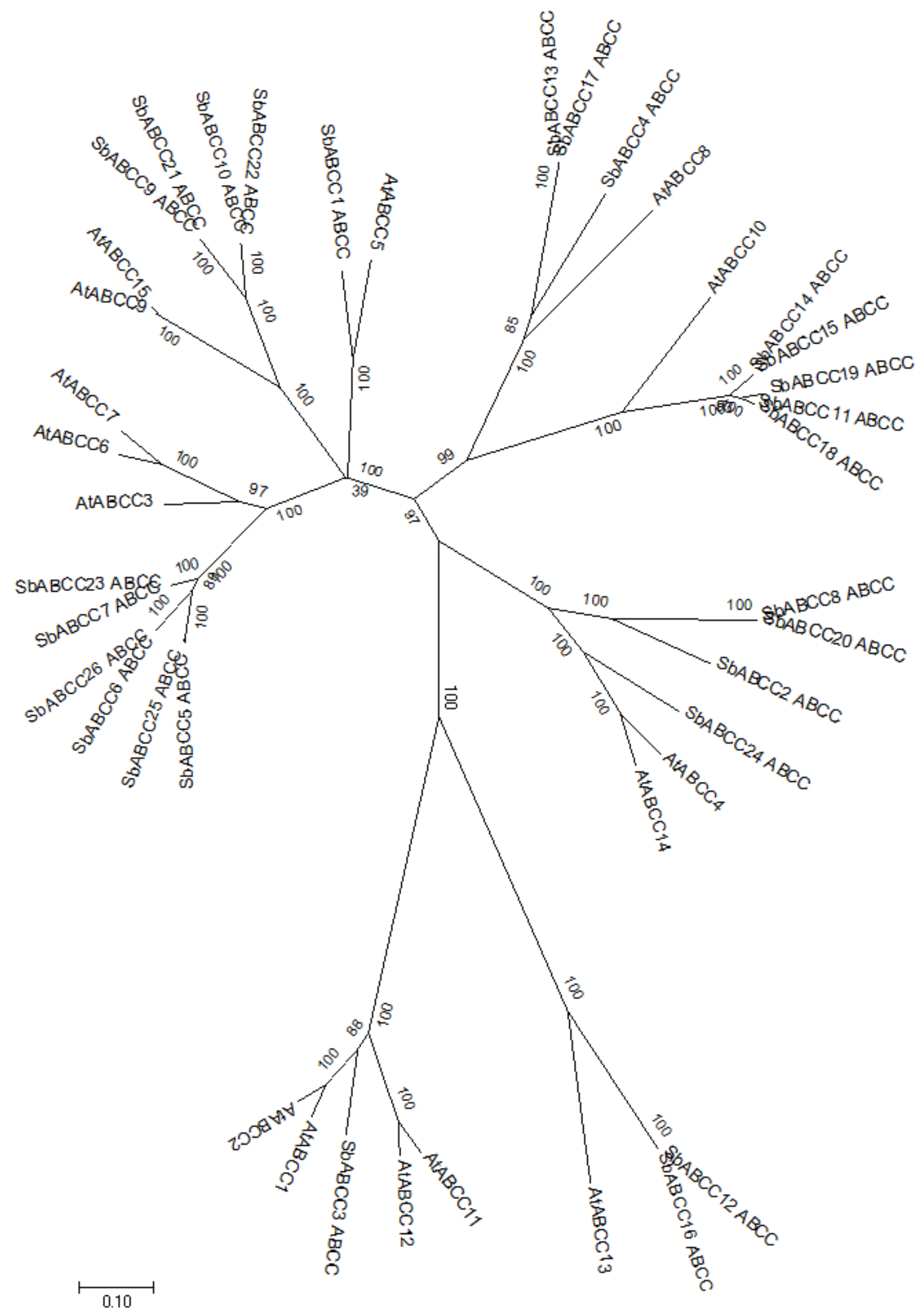

Figure 3: P Phylogenetic tree of ABCC proteins from S. bicolor and A. thaliana AtABCCn, A. thaliana ABCC proteins; SbABCCn, S. bicolor ABCC proteins. n: Number

S-conjugate and are involved in the regulation of stomatal aperture as a pump for folates with a high capacity. This extrapolation stems from the functions of AtABCC14 and AtABCC4 in pumping of glutathione S-conjugate and the involvement of AtABC4 (alias AtMRP4) in regulating stomatal aperture as a pump for folates with high capacity $[59,60]$. 
At $\mathrm{ABCC} 1$ and AtABCC2 are pumps for glutathione S-conjugates and they mediate the transport of S-conjugates including GSH, S-(2,4-dinitrophenyl)-glutathione (DNP-GS), GSSG, cyaniding 3-glucooside-GS (C3G-GS) and metolachlor-GS (MOCGS), glucuronides such as 17-beta-estradiol 17-(beta-D-glucuronide) (E(2)17betaG), and the chlorophyll catabolite including Brassica napus nonfluorescent chlorophyll catabolite (Bn-NCC-1) [61]. Thus the formation of clusters of these A. thaliana ABCC transporters with SbABCC3 suggests that the S. bicolor ABC transporter carries out these functions.

\section{Phylogeny of Sorghum bicolor and Arabidopsis thaliana ABCD Subfamilies}

The result from phylogeneitc analysis of ABCD subfamily proteins from Sorghum bicolor and Arabidopsis thaliana is presented in Figure 4. The ABCD transporter proteins from A. thaliana (AtABCD1 and AtABCD2) did not cluster closely with the proteins from S. bicolor with bootstrap values ranging from 0 to 100. This is might be due to marked differences between their trans membrane domain (TMD) sequences. For this reason, the $S$. bicolor ABCD transporters will most likely differ in their functions as regards the substrates that are transported.

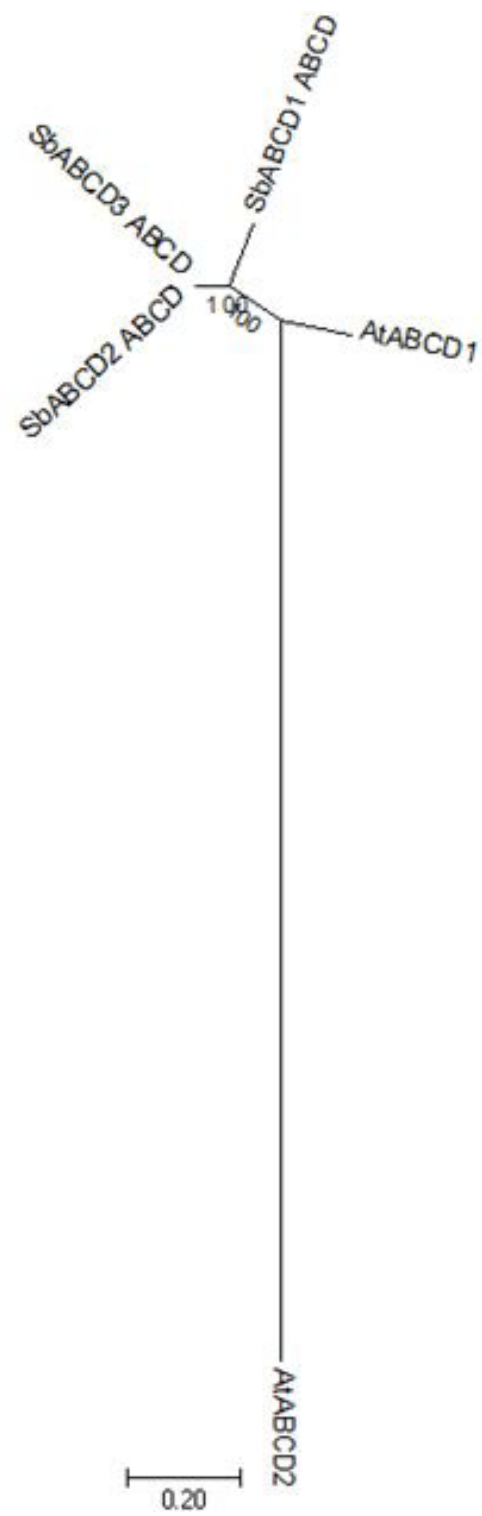

Figure 4: Phylogenetic tree of $\mathrm{ABCD}$ proteins from S. bicolor and A. thaliana AtABCDn, A. thaliana ABCD proteins; SbABCDn, S. bicolor ABCD proteins. n: Number

\section{Phylogeny of Sorghum bicolor and Arabidopsis thaliana ABCE and ABCF Subfamilies}

The result for phylogenetic analysis of the soluble transport proteins (ABCE and ABCF subfamilies) from A. thaliana and S. bicolor is shown in Figure 5. Four cluster groups were formed by the protein sequences with bootstrap values of 87 to 100 . One cluster group consisted of SbABCE2, AtABCF5, and AtABCF2. SbABCF1 clustered with SbABCE4, SbABCE1, and SbABCE3. The cluster formed between SbABCE2, AtABCE5, and AtABCF2 (Figure 5) cannot be used to deduce the functions of the Soghum bicolor $\mathrm{ABCE}$ transporters because the proteins from A. thaliana have not been empirically characterized. 


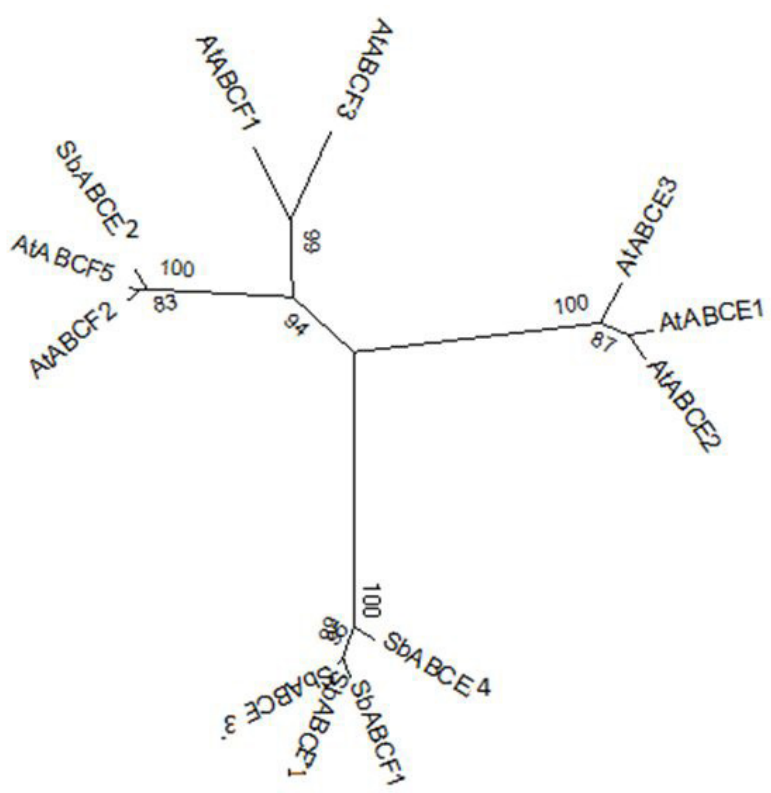

Figure 5: Phylogenetic tree of $\mathrm{ABCE}$ and $\mathrm{ABCF}$ proteins from

S. bicolor and A. thaliana AtABCEn, A. thaliana ABCE proteins;

SbABCEn, S. bicolor ABCE proteins. n: Number AtABCFn, A. thaliana

$\mathrm{ABCF}$ proteins; SbABCFn, S. bicolor ABCF proteins

\section{Phylogeny of Sorghum bicolor and Arabidopsis thaliana ABCI Subfamilies}

The result for phylogenetic analysis of $\mathrm{ABC}$ transporters of the ABCI subfamily from A. thaliana and S. bicolor is shown in Figure 6. Four cluster groups were formed between ABCI transport sequences from both plants. AtABCI2, AtABCI16, AtABCI15, and AtABCI10 clustered closely with SbABCI22. Similarly, AtABCI12, AtABCI3, and AtABCI17 formed a cluster group with SbABCI21, SbABCI18, SbABCI6, and SbABCI10. In addition, AtABCI13, AtABCI11, and AtABCI7 grouped together with SbABCI1. AtABCI1, AtABCI6, AtABCI8, and AtABCI9 were tightly clustered with SbABCI3.

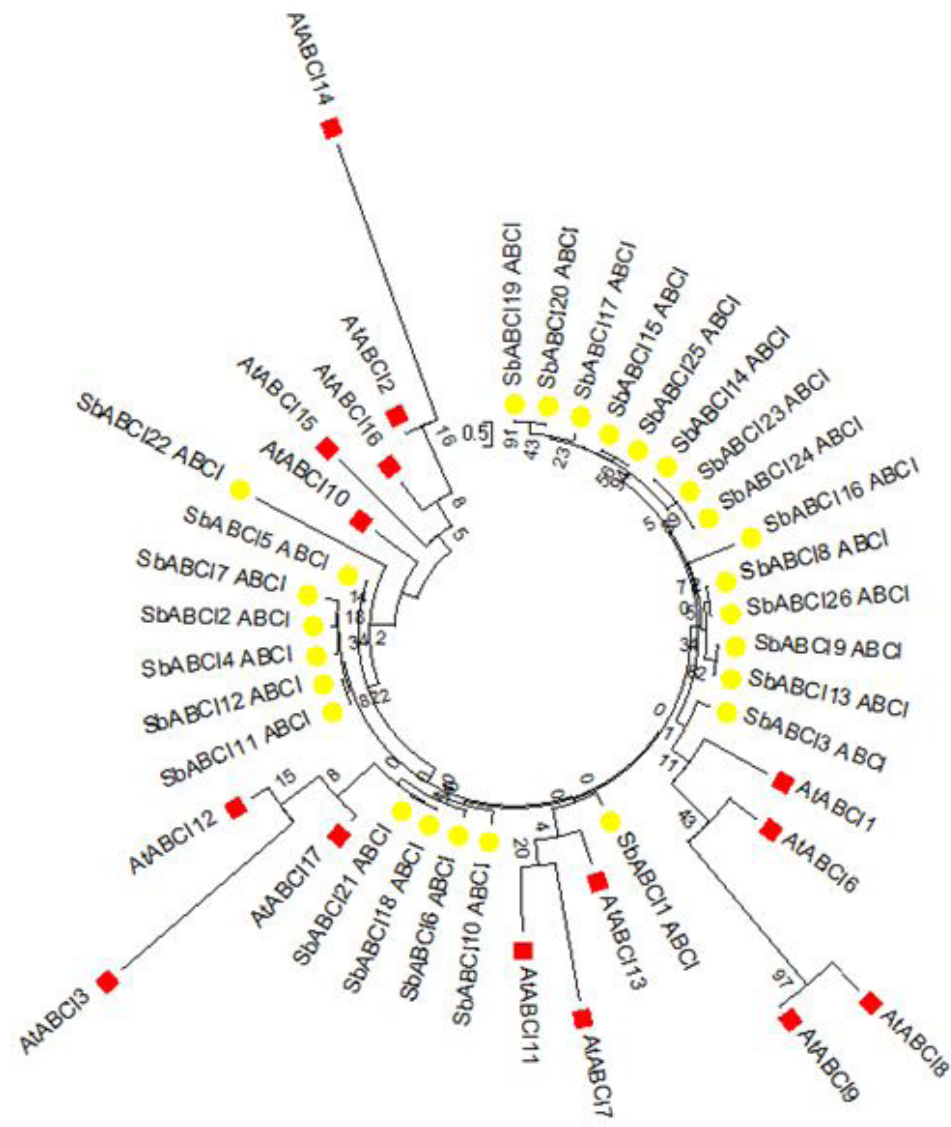

Figure 6: Phylogenetic tree of ABCI proteins from S. bicolor and A. thaliana OS. bicolor ABCI proteins $\square$ A. thaliana ABCI proteins 
The divergence of members of the ABCI subfamily is too large making it difficult for their phylogenetic relationships to be resolved [36]. Thus tight clustering of members of the subfamily from S. bicolor and A. thaliana was not observed (Figure 6). Nevertheless AtABCI3 seemed to cluster with SbABCI21, SbABCI18, SbABCI6 and SbABCI10; therefore they may have involvements in the export of heme to the mitochondrion for the biogenesis of c-type cytochromes [36]. Thus the S. bicolor ABC proteins could, with caution, be inferred to be involved in this function. In the same vein, SbABCI3 could cautiously be inferred to be playing a certain part in the biogenesis of c-type cytochrome because it clustered with AtABCI1 (alias cytochrome c biogenesis ABC export protein ccmA-like).

\section{Analysis of Sequences Orthologous to Sorghum bicolor ABC Transporters}

The identification of orthologs of Sorghum bicolor ABC transporters in maize and thale cress provides genetic and molecular resources for the elucidation of the biological and physiological functions of $\mathrm{ABC}$ transporters in sorghum. This ultimately gives an opportunity for development of better cultivars of the crop. Several orthologs to S. bicolor ABC transporters were detected but only those whose functions have been experimentally determined were used for inferring the functions of S. bicolor ABC transporters (Table S2). But it is pertinent to note that members of ABC transporter superfamily have in common the functions of active transport of substrates across membranes and hydrolysis of adenosine triphosphate (ATP) [34]. In other words they carry out ATPase activity, coupled to transmembrane movement of substances.

SbABCB5 and SbABCB8 are inferred to carry out auxin efflux transmembrane transporter activities and SbABCC1 is likely to function in sulfonylurea receptor activity (Table S2). SbABCC1 is also predicted to show response to stress and cellular potassium ion homeostasis (Table S2; put citation here). In addition, SbABCI8, SbABCI26, and SORBI_006G155700 will most likely function in fatty acid transport activities (Table S2). One member of the ABCB subfamily, SbABCB25 (P-glycoprotein 1), has been characterized; it functions in calmudolin binding and auxin efflux transmembrane transporter activity.

Transport proteins planted within membranes are major targets for ameliorating the efficiency with which plants obtain and utilise water and nutrients [62]. Transport proteins are also key players in the mechanisms by which plants tolerate adverse environments including saline and acid soils. Acidic soils contain $\mathrm{Al}^{3+}$ ions that cause damage to the root tips of susceptible plants thereby inhibiting root growth by impaired uptake of nutrients and water [62]. Genetic studies in Sorghum bicolor have identified markers linked to $\mathrm{Al}^{3+}$-tolerant alleles of the $\mathrm{S}$. bicolor multidrug and toxin extrusion (MATE) gene SbMATE [63]. These markers have been applied by sorghum breeders to introgress the most favourable SbMATE alleles into germplasm of sorghum. The identification of AtABCB27, which is involved in response to aluminium, as an ortholog to SbABCB1 and SbABCB6 adds more genetic bases to the study of $\mathrm{Al}^{3+}$ tolerance of sorghum and provides opportunity for increasing the genetic scope of breeding cultivars that are tolerant to $\mathrm{Al}^{3+}$ (Table S2). It is worthy of note to state that the result for orthology analysis of SbABCB1 agrees with the result for phylogenetic anlaysis which showed that SbABCB1 clustered tightly with AtABCB27 (Figure 2) (Table S2).

The functions of SbABCB5 and SbABCB8 were inferred as auxin efflux transmembrane transport based on their homology to AtABCB19 and AtABCB1 (alias AtPGP1) (Table S2). This agrees with the results from phylogenetic analysis which showed clustering of SbABCB5 with AtABCB19 and SbABCB8 with AtABCB1 (Figure 2). Auxin is a key player in regulation of plant growth and development; it orchestrates division, elongation, and differentiation of cells, development of embryo, tropisms in root and stem, dominance in apices, and transition to flowering [64]. Knowing that auxin transport proteins are involved in the regulation of intracellular and cell to cell auxin fluxes, the identification of SbABCB5 and SbABCB8 in sorghum genome could help in giving insight into the role of auxin in sorghum and monocots in general.

SbABCB8 also has a promising function in calmodulin binding (Table S2). Genetic and molecular studies of the function and mechanism of action of the protein and its corresponding gene could therefore give insight into nonhost resistance, the most prevalent form of immunity in plants, in sorghum. This is because calmodulin has been implicated as a crucial requirement for nonhost resistance in $A$. thaliana [65].

Xenobiotics are a defense mechanism that describes the metabolism of organic compounds with abiotic origin [66]. In other words, it is a biotransformation mechanism and an essential part of plants' strategy to cope with the potentially negative impacts of xenobiotics on growth and development. The mechanism is especially evident in the ability of plants to metabolically detoxify herbicides. There is however a differential ability of plant species to carry out this metabolic task; allowing for its extensive exploitation in modern day agriculture by use of selective herbicides that are not harmful to the crop but harmful to the associated weeds. Selective herbicidal activities come about by compounds collectively known as "herbicide safeners", which consist of chemically many and different compounds that possess the ability to protect grass crops from injury due to herbicide application without lowering activity of herbicide in reducing target species of weeds [67]. Notwithstanding their widespread use, the molecular mechanisms employed by safeners in inducing their corresponding genes or signaling pathways are not known. Thus the identification of SbABCB9 as having a promising function in xenobiotic-transporting ATPase activity could help to uncover the molecular mechanism of induction of the corresponding gene or signaling pathways in sorghum protecting themselves from xenobiotics-based damages (Table S2). Furthermore, protection of S. bicolor from herbicide-based damages could be achieved by increasing the expression of SbABCB4 genes. 
The identification of maize TPA (alias lpa 1 ) as an ortholog to SbABCC1 is an indication that the protein is involved in accumulation of phytic acid in seed grains of sorghum (Table S2). The corresponding gene of SbABCC1 could be manipulated using a sitespecific technology like clustered regularly interspaced short palindromic repeats and its associated protein (CRISPR/Cas9) to down regulate or delete a segment of the gene that will cause a reduction of phytic acid in the grains. Shi et al. [27]. demonstrated that targeting the gene responsible for seed/grain phytic acid accumulation in immature embryo can result in embryo-specific and eventually seed- or grain-specific phytic acid reduction. Circumventing the problem of high phytic acid content in some grains of sorghum and even in grains/seeds of other cereals and legumes by site-specific embryo-specific phytic acid reduction targeting only the gene responsible for loading of the acid into grains/seeds could be phenomenal. This is due especially to the fact that a reduction of grain/seed phytic acid that has been attempted by editing other genes in the acid biosynthetic pathway have resulted in crops with overall reduced vigour, stunted growth, reduced seed viability, and reduced yield $[27,68]$.

Furthermore, SbABCC1 showed homology to inositol hexakisphosphate transporter of $A$. thaliana (AtABCC5) which agrees with the results for phylogenetic analysis with SbABCC1 forming a cluster group with AtABCC5 (Figure 3) (Table S2). AtABCC5 functions in sulfonylurea receptor activity and shows response to salt stress via cellular potassium ion homeostasis. Thus genetic and molecular studies of the functions of SbABCC1 and its corresponding genes could help in breeding for salt tolerance in sorghum.

The results for orthology and phylogenetic analysis as regards SbABCC3 and AtABCC2 are in agreement (Table S2) (Figure 3). AtABCC2 is involved in response to cyclopentenone. Cyclopentenone take part in the biosynthesis of jasmonic acid, a defense signal employed by plants during stress [69]. The implication is that the role of cyclopentenone signals in sorghum defense against biotic and abiotic stress could be uncovered.

The functions of SbABCI8, SbABCI26, and SORBI_006G155700 are inferred from their ortholog AtABCG11, which carries out functions in fatty acid transport and has involvement in cutin transport, fatty acid transport (Table S2). The homology of sorghum $\mathrm{ABCI}$ proteins to thale cress ABCG proteins buttresses the difficulty in resolving the phylogenetic relationships of the ABCI subfamily due to enormous diversity [36]. Cutin is a fatty substance that is deposited in cell walls and outer protective tissues of the plant body. Cutin is therefore one of the first line of defense in plants. With the identification of SbABCI8, SbABCI26, and SORBI_006G155700 as fatty acid transporters in cutin formation, the mechanism and possible exploitation of cutin formation for defense could be exploited to better sorghum cultivars.

\section{Conclusion}

$\mathrm{ABC}$ transporters play essential role in plant organogenesis, nutrition, development, response to biotic and abiotic stress, and interaction with its environment. Thus, this study which involved an inventory of Sorghum bicolor ABC transporters could be a launch pad for unraveling the role of the proteins and corresponding genes in key biological processes in Sorghum bicolor. Therefore, there is need for further research to be conducted for elucidation of the biological and physiological functions of ABC transporters from Sorghum bicolor. Such studies could ultimately open up opportunity for improvement of Sorghum bicolor through tweaking of $\mathrm{ABC}$ transporter genes which are involved in organogenesis, nutrition, development, response to biotic and abiotic stress, and interaction with environment.

\section{Supplementary Info}

\section{References}

1. Gautam JC (2008) Country Report on the State of Plant Genetic Resources for Food and Agriculture.

2. Dean M, Rzhetsky A, Allikmets R (2001) The Human ATP-Binding Cassette transporter superfamily. Genome Res 11: $1007-17$.

3. Hwang JU, Song WY, Hong D, Ko D, Yamaoka Y, Jang S, et al. (2016) Plant ABC Transporters Enable Many Unique Aspects of a Terrestrial Plant's Lifestyle. Mol Plant 9: 338-55.

4. Pighin JA, Zheng H, Balakshin LJ, Goodman IP, Western TL, et al. (2004) Plant Cuticular Lipid Export Requires an ABC Transporter. Science 306: 702-4.

5. Suh MC, Samuels AL, Jetter R, Kunst L, Pollard M, et al. (2005) Cuticular Lipid Composition, Surface Structure, and Gene Expression in Arabidopsis Stem Epidermis. Plant Physiol 139: 1649-65.

6. Bird D, Beisson F, Brigham A, Shin J, Greer S, et al. (2007) Characterization of Arabidopsis ABCG11/WBC11, an ATP binding cassette (ABC) transporter that is required for cuticular lipid secretion. Plant J. 52, 485-98.

7. Sánchez-Fernández R, Davies TG, Coleman JO, Rea PA (2001) The Arabidopsis thaliana ABC protein superfamily, a complete inventory. J Biol Chem 276: 30231-44.

8. Panikashvili D, Shi JX, Schreiber L, Aharoni A (2011) The Arabidopsis ABCG13 transporter is required for flower cuticle secretion and patterning of the petal epidermis. New Phytol 190: 113-24.

9. Quilichini TD, Douglas CJ, Samuels AL (2014) New views of tapetum ultrastructure and pollen exine development in Arabidopsis thaliana. Ann Bot 114: 1189201 .

10. Niu BX, He FR, He M, Ren D, Chen LT, et al. (2013) The ATP-binding Cassette Transporter OsABCG15 is Required for Anther Development and Pollen Fertility in Rice. J Integr Plant Biol 55: 710-20.

11. Wu L, Guan Y, Wu Z, Yang K, Lv J, et al. (2014) OsABCG15 encodes a membrane protein that plays an important role in anther cuticle and pollen exine formation in rice. Plant Cell Rep 33: 188-99. 
12. Choi H, Ohyama K, Kim YY, Jin JY, Lee SB, et al. (2014) The role of Arabidopsis ABCG9 and ABCG31 ATP binding cassette transporters in pollen fitness and the deposition of steryl glycosides on the pollen coat. Plant Cell 26: 310-24.

13. Crouzet J, Roland J, Peeters E, Trombik T, Ducos E, et al. (2013) NtPDR1, a plasma membrane ABC transporter from Nicotiana tabacum, is involved in diterpene transport. Plant Mol. Biol 82: 181-92.

14. Stukkens Y, Bultreys A, Grec S, Trombik T, Vanham D, et al. (2005) NpPDR1, a Pleiotropic Drug Resistance-Type ATP-Binding Cassette Transporter from Nicotiana plumbaginifolia, Plays a Major Role in Plant Pathogen Defense. Plant Physiol 139: 341-52.

15. Yazaki K, Shitan N, Takamatsu H, Ueda K, Sato F (2001) A novel Coptis japonica multidrug-resistant protein preferentially expressed in the alkaloid-accumulating rhizome. J Exp Bot 52: 877-9.

16. Shitan N, Bazin I, Dan K, Obata K, Kigawa K, et al. (2003) Involvement of CjMDR1, a plant multidrug-resistance-type ATP-binding cassette protein, in alkaloid transport in Coptis japonica. Natl Acad Sci 100: 751 LP-756.

17. Ko D, Kang J, Kiba T, Park J, Kojima M, et al. (2014) Arabidopsis ABCG14 is essential for the root-to-shoot translocation of cytokinin. Proc Natl Acad Sci 111: $7150-5$.

18. Kuromori T, Miyaji T, Yabuuchi H, Shimizu H, Sugimoto E, et al. (2010) ABC transporter AtABCG25 is involved in abscisic acid transport and responses. Proc Natl Acad Sci 107: 2361-6.

19. Kang J, Hwang JU, Lee M, Kim YY, Assmann SM, et al. (2010) PDR-type ABC transporter mediates cellular uptake of the phytohormone abscisic acid. Proc. Natl Acad Sci 107: 2355-60.

20. Kang J, Park J, Choi H, Burla B, Kretzschmar T,et al. (2011) Plant ABC Transporters. Arabidopsis Book 9: e0153.

21. Geisler M, Blakeslee JJ, Bouchard R, Lee OR, Vincenzetti V, et al. (2005) Cellular efflux of auxin catalyzed by the Arabidopsis MDR/PGP transporter AtPGP1. Plant J 44: 179-94.

22. Yang L, Li G, Zhang J, Jiang D, Chen W (2011) Compatibility of Coniothyrium minitans with compound fertilizer in suppression of Sclerotinia sclerotiorum. Biol Control 59: 221-7.

23. Kamimoto Y, Terasaka K, Hamamoto M, Takanashi K, Fukuda S, et al. (2012) Arabidopsis ABCB21 is a Facultative Auxin Importer/Exporter Regulated by Cytoplasmic Auxin Concentration. Plant Cell Physiol 53: 2090-100.

24. Terasaka K, Blakeslee JJ, Titapiwatanakun B, Peer WA, Bandyopadhyay A, et al. (2005) PGP4, an ATP binding cassette P-glycoprotein, catalyzes auxin transport in Arabidopsis thaliana roots. Plant Cell 17: 2922-39.

25. Sasse J, Simon S, Gübeli C, Liu GW, Cheng X, et al. (2018) Asymmetric Localizations of the ABC Transporter PaPDR1 Trace Paths of Directional Strigolactone Transport. Curr Biol 25: 647-55.

26. Zolman BK, Silva ID, Bartel B (2001) The Arabidopsis pxa1 mutant is defective in an ATP-binding cassette transporter-like protein required for peroxisomal fatty acid beta-oxidation. Plant Physiol 127: 1266-78.

27. Shi J, Wang H, Schellin K, Li B, Faller M, et al. (2007) Embryo-specific silencing of a transporter reduces phytic acid content of maize and soybean seeds. Nat Biotechnol 25: 930-7.

28. Song WY, Park J, Mendoza-Cózatl DG, Suter-Grotemeyer M, Shim D, et al. (2010) Arsenic tolerance in Arabidopsis is mediated by two ABCC-type phytochelatin transporters. Proc Natl Acad Sci 107: 21187-92.

29. Park J, Song WY, Ko D, Eom Y, Hansen TH, et al. (2011) The phytochelatin transporters AtABCC1 and AtABCC2 mediate tolerance to cadmium and mercury. Plant J 69: 278-88.

30. Xu C, Fan J, Riekhof W, Froehlich JE, Benning C (2003) A permease-like protein involved in ER to thylakoid lipid transfer in Arabidopsis. EMBO J 22: 2370-9. 31. Lu B, Benning C (2009) A 25-Amino Acid Sequence of the Arabidopsis TGD2 Protein Is Sufficient for Specific Binding of Phosphatidic Acid. J Biol Chem 284: $17420-7$

32. Awai K, Xu C, Lu B, Benning C (2006) Lipid trafficking between the endoplasmic reticulum and the chloroplast. Biochem Soc Trans 34: 395-8.

33. Kim S, Yamaoka Y, Ono H, Kim H, Shim D, et al. (2013) AtABCA9 transporter supplies fatty acids for lipid synthesis to the endoplasmic reticulum. Proc Natl Acad Sci 110: 773-8.

34. Davidson AL, Dassa E, Orelle C, Chen J (2008) Structure, function, and evolution of bacterial ATP-binding cassette systems. Microbiol Mol Bio Rev 72: 317-64. 35. Andolfo G, Ruocco M, Donato AD, Frusciante L, Lorito M, et al. (2015) Genetic variability and evolutionary diversification of membrane ABC transporters in plants. BMC Plant Biol 15: 51.

36. Verrier PJ, Bird D, Burla B, Dassa E, Forestier C, et al. (2008) Plant ABC proteins - a unified nomenclature and updated inventory. Trends Plant Sci 13: 151-9. 37. Garcia O, Bouige P, Forestier C, Dassa E (2004) Inventory and comparative analysis of rice and Arabidopsis ATP-binding cassette (ABC) systems. J Mol Biol 343: 249-65.

38. Multani DS, Briggs SP, Chamberlin MA, Blakeslee JJ, Murphy AS, et al. (2003) Loss of an MDR transporter in compact stalks of maize br2 and sorghum dw3 mutants. Science 302: 81-4.

39. Paterson AH, Bowers JE, Bruggmann R, Dubchak I, Grimwood J, et al. (2009) The Sorghum bicolor genome and the diversification of grasses. Nature 457: 551-6. 40. Altschul SF, Madden TL, Schäffer AA, Zhang J, Zhang Z, et al. (1997) Gapped BLAST and PSI-BLAST: a new generation of protein database search programs. Nucleic Acids Res 25: 3389-402 (1997).

41. Altschul SF, Koonin EV (2018) Iterated profile searches with PSI-BLAST\&\#x2014;a tool for discovery in protein databases. Trends Biochem Sci 23: 444-7.

42. Sigrist CJ, Cerutti L, Hulo N, Gattiker A, Falquet L, et al. (2002) PROSITE: A documented database using patterns and profiles as motif descriptors. Brief Bioinform 3: 265-74.

43. Sigrist CJ, de Castro E, Cerutti L, Cuche BA, Hulo N, et al. (2013) New and continuing developments at PROSITE. Nucleic Acids Res. 41, D344-7.

44. Krogh A, Larsson B, von Heijne G, Sonnhammer EL (2001) Predicting transmembrane protein topology with a hidden markov model: application to complete genomes11Edited by F. Cohen. J Mol Biol 305: 567-80.

45. Gray KA, Yates B, Seal RL, Wright MW, Bruford EA (2015) Genenames.org: the HGNC resources in 2015. Nucleic Acids Res 43: D1079-85 (2015).

46. Cole SP, Bhardwaj G, Gerlach JH, Mackie JE, Grant CE, et al. (1992) Overexpression of a transporter gene in a multidrug-resistant human lung cancer cell line. Science 258: 1650-4.

47. Muhrez K, Largeau B, Emond P, Montigny F, Halimi JM, et al. (2017) Single nucleotide polymorphisms of ABCC2 modulate renal secretion of endogenous organic anions. Biochem Pharmacol 140: 124-38. 
48. McIlwain SJ, Peris D, Sardi M, Moskvin OV, Zhan F, et al. (2016) Genome Sequence and Analysis of a Stress-Tolerant, Wild-Derived Strain of Saccharomyces cerevisiae Used in Biofuels Research. G3 6: 1757-66.

49. Goffeau A, Barrell BG, Bussey H, Davis RW, Dujon B, et al. (1996) Life with 6000 Genes. Science. 274: 546-567.

50. Thompson JD, Higgins DG, Gibson TJ (1994) CLUSTAL W: Improving the sensitivity of progressive multiple sequence alignment through sequence weighting, position-specific gap penalties and weight matrix choice. Nucleic Acids Res 22: 4673-80.

51. Kumar S, Stecher G, Tamura K (2016) MEGA7: Molecular Evolutionary Genetics Analysis version 7.0 for bigger datasets. Mol Biol Evol 33: 1870-4.

52. Stamatakis A (2014) RAxML version 8: a tool for phylogenetic analysis and post-analysis of large phylogenies. Bioinformatics 30, $1312-3$.

53. Çakır B, Kılıçkaya O (2013) Whole-genome survey of the putative ATP-binding cassette transporter family genes in Vitis vinifera. PLoS One 8: e78860.

54. Vasiliou V, Vasiliou K, Nebert DW (2009) Human ATP-binding cassette ( ABC ) transporter family. Hum Genomics 3: 281-90.

55. Venter JC, Adams MD, Myers EW, Li PW, Mural RJ, et al. (2001) The sequence of the human genome. Science 291: $1304-51$.

56. Theodoulou F, Kerr I (2015) ABC transporter research: going strong 40 years on. Biochem. Soc Trans. 43: 1033-40.

57. Sugiyama A, Shitan N, Sato S, Nakamura Y, Tabata S, et al. (2006) Genome-wide analysis of ATP-binding cassette (ABC) proteins in a model legume plant, Lotus japonicus: Comparison with Arabidopsis ABC protein family. DNA Res 13: 205-28.

58. Noh B, Murphy AS, Spalding EP (2001) Multidrug Resistance-like Genes of Arabidopsis Required for Auxin Transport and Auxin-Mediated Development. Plant Cell 13: 2441-54.

59. Martinoia E, Klein M, Geisler M, Bovet L, Forestier C, E. et al. (2002) Multifunctionality of plant ABC transporters - More than just detoxifiers. Planta 214, 345-55.

60. Klein M, Geisler M, Suh SJ, Kolukisaoglu HU, Azevedo L, et al. (2004) Disruption of AtMRP4, a guard cell plasma membrane ABCC-type ABC transporter, leads to deregulation of stomatal opening and increased drought susceptibility. Plant J 39: 219-36.

61. Kolukisaoglu HU, Bovet L, Klein M, Eggmann T, Geisler M, et al. (2002) Family business: the multidrug-resistance related protein (MRP) ABC transporter genes in Arabidopsis thaliana. Planta 216: 107-19.

62. Schroeder JI, Delhaize E, Frommer WB, Guerinot ML, Harrison MJ, et al. (2013) Using membrane transporters to improve crops for sustainable food production. Nature 497: 60-6.

63. Magalhaes JV, Liu J, Guimarães CT, Lana UG, Alves VM, et al. (2007) A gene in the multidrug and toxic compound extrusion (MATE) family confers aluminum tolerance in sorghum. Nat Genet 39: 1156-61.

64. Balzan S, Johal GS, Carraro N (2014) Plant Science The role of auxin transporters in monocots development. Front Plant Sci 5: 393.

65. Campe R, Langenbach C, Leissing F, Popescu GV, Popescu SC, et al. (2016) ABC transporter PEN3/PDR8/ABCG36 interacts with calmodulin that, like PEN3, is required for Arabidopsis nonhost resistance. New Phytol 209, 294-306.

66. Riechers DE, Kreuz K, Zhang Q (2010) Detoxification without intoxication: herbicide safeners activate plant defense gene expression. Plant Physiol. 153: 3-13. 67. Hatzios KK, Burgos N (2004) Metabolism-Based Herbicide Resistance: Regulation by Safeners. Weed Sci 52: $454-67$.

68. Liang Z, Zhang K, Chen K, Gao C (2014) Targeted Mutagenesis in Zea mays Using TALENs and the CRISPR/Cas System. J Genet Genomics 41: 63-8. 69. Howe GA (2001) Cyclopentenone signals for plant defense: remodeling the jasmonic acid response. Proc. Natl. Acad. Sci U S A 98: 12317-9.

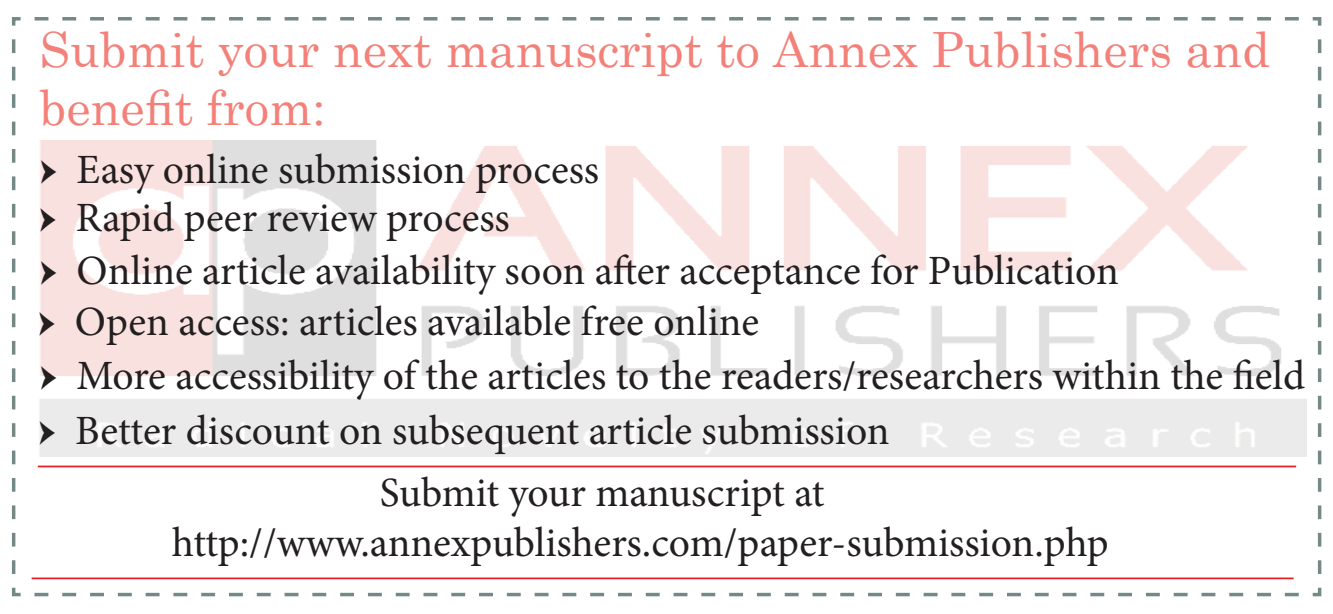

